\title{
Metaphyseal dysplasia, Braun-Tinschert type
}

INSERM

\section{Source}

INSERM. (1999). Orphanet: an online rare disease and orphan drug data base. Metaphyseal dysplasia, Braun-Tinschert type. ORPHA:85188

Metaphyseal dysplasia, Braun-Tinschert type is characterised by metapyhseal undermodeling with broadening of the long bones and femora with an 'Erlenmeyer flask" appearance, expansion and bowing of the radii with severe varus deformity and flat exostoses of the long bones at the metadiaphyseal junctions. 